\title{
Screening for FMR-1 premutations in 122 older Flemish males presenting with ataxia
}

\author{
Hilde Van Esch*, ${ }^{*}$, Rene Dom ${ }^{2}$, Dorien Bex ${ }^{1}$, Ivo Salden ${ }^{1}$, Jo Caeckebeke ${ }^{3}$, Alain Wibail ${ }^{4}$, \\ Martine Borghgraef ${ }^{1}$, Eric Legius ${ }^{1}$, Jean-Pierre Fryns ${ }^{1}$ and Gert Matthijs ${ }^{1}$
}

${ }^{1}$ Centre for Human Genetics, University Hospital Leuven, Leuven, Belgium; ${ }^{2}$ Department of Neurology, University Hospital Leuven, Leuven, Belgium; ${ }^{3}$ Onze-Lieve-Vrouw Ziekenhuis, Aalst, Belgium; ${ }^{4}$ Ziekenhuis Oost-Limburg, Genk, Belgium

Recently, Hagerman et al described the occurrence of a late-onset neurological disorder in five male carriers of the fragile-X (FMR-1) premutation. The major characteristics of this disorder, designated the Fragile-X Tremor Ataxia Syndrome (FXTAS), are progressive intention tremor, cerebellar ataxia and cognitive decline. Most cases of FXTAS published thus far were ascertained through families with a known fragile-X proband. Since cerebellar ataxia is one of the main cardinal features, we performed FMR-1 premutation screening in 122 male patients, older than 50 years, who were referred to us for testing of the spinocerebellar ataxia (SCA 1, 2, 3, 6, 7) genes and who were found to be negative. In this group of patients, we found five patients with an FMR-1 premutation. In four of them, a definite diagnosis of FXTAS could be made, based on the proposed diagnostic clinical and radiological criteria for FXTAS. In light of these figures, we recommend that FMR-1 analysis should be included in the molecular diagnostic work-up in the group of male ataxia patients older than $\mathbf{5 0}$ years.

European Journal of Human Genetics (2005) 13, $121-123$. doi:10.1038/sj.ejhg.5201312

Published online 13 October 2004

Keywords: FMR-1; premutation; FXTAS; ataxia

In 2001, Hagerman et al ${ }^{1}$ described the occurrence of a lateonset neurological disorder in five male carriers of the fragile-X (FMR-1) premutation. This disorder, designated the Fragile-X Tremor Ataxia Syndrome (FXTAS), consists of progressive intention tremor, cerebellar ataxia and cognitive decline. Since the first description additional symptoms were documented, including parkinsonism, peripheral neuropathy, autonomic dysfunction, lowerlimb proximal muscle weakness and behavioral difficulties. Based on a series of 26 patients, diagnostic criteria for FXTAS have been proposed recently by Jacquemont et al. ${ }^{2}$ The presence of symmetrical regions of increased $\mathrm{T} 2$ signal

*Correspondence: Dr H Van Esch, Centre for Human Genetics, University Hospital Gasthuisberg, Herestraat 49, 3000 Leuven, Belgium. Tel.: + 3216-345903; Fax: +32-16-346051; E-mail: Hilde.Vanesch@med.kuleuven.ac.be

Received 8 July 2004; revised 19 August 2004; accepted 31 August 2004 intensity in the middle cerebellar peduncles (MCP) and adjacent white matter is thought to be a specific sign and included as radiological inclusion criterion for this syndrome. Data of a large survey have demonstrated an agerelated penetrance of the combination of reported tremor and ataxia in male premutation carriers, going up to $47 \%$ in the group of 70-79 years. $^{3}$

Most cases of FXTAS published thus far were ascertained through families with a known fragile-X proband. Since cerebellar ataxia is one of the main cardinal features, we decided to perform fragile $\mathrm{X}$ premutation screening in male patients, who were referred to our laboratory for testing of the spinocerebellar ataxia (SCA) genes and who were found to be negative for the expansion. We retrospectively selected all male probands with ataxia, who were 50 years or older at the time of referral. Of 137 patients, 15 $(10.9 \%)$ were found positive for an (CAG) $n$ expansion in one of the SCA genes. Of the remaining 122 men, all were 
negative for the expansion in the $S C A 1,2,3,6$ and 7 genes. Among these 122 patients, we have found five (4.1\%) with a CGG repeat number in the FMR-1 gene above 50. Only one of these patients was known to the clinical geneticists as a normal transmitting male; however, this was not known to the laboratory were the SCA analysis was performed on request of the neurologist.

Subsequently, we reviewed the clinical records and brain imaging results of the five identified patients. Two of them were deceased since their initial referral, the other three were neurologically re-evaluated by a neurologist and a clinical geneticist. Patient 1 developed severe bilateral intention tremor and sensory symptoms in his upper limbs at the age of 62 years. In the following years, dysarthria and cerebellar ataxia were noted, resulting in frequent falling. At that time, he also developed severe bowel and bladder incontinence, worsening his social isolation. Since the age of 77 years, he is wheelchair bound and stays in a nursing home. Neurological re-examination at the age of 80 years included an intention tremor (left $>$ right) in the upper limbs, dysarthria and dysmetria in all four extremities. He has weak to absent deep-tendon reflexes, upgaze nystagmus and saccadic eye movements. He does not recall recent events, but long-term memory seems to be conserved. A first MRI-scan, performed at the age of 65 years already showed the white matter lesions in the MCP, together with important cortical and ponto-cerebellar atrophy. A further progression of these lesions was seen on a follow-up MRI 10 years later. This patient was shown to have 91 CGG repeats. Patient 2 was seen by a neurologist 1 year ago, at the age of 61 years, with an acute pyramidal syndrome consisting of transient paresis of the left lower limb with subsequent partial recuperation, probably due to a transient ischemic attack. Before this event, he had complaints about dysequilibrium and erectile dysfunction. Owing to the presence of mild ataxia, he was referred for molecular SCA diagnosis. At recent re-evaluation at the age of 62 years, he still presents a left pyramidal syndrome together with a bilateral discrete intention tremor and mild ataxia. Electromyography showed signs of a bilateral demyelinating sensori-motor polyneuropathy. MRI scan showed atrophy of the fronto-parietal cortex, atrophy of the splenium of the corpus callosum and periventricular leucomalacia together with the typical lesions in the MPC and adjacent white matter. This patient was shown to carry 80 repeats. Patient 3 reported onset of tremor and unsteadiness at the age of 64 years, together with complaints of memory loss. During the subsequent years, the cerebellar symptoms worsened, as well as the cognitive decline. Now at the age of 71 years, he is wheelchair bound and nursed in a home for demented elderly. Eye movements are saccadic without nystagmus. He presents bradykinesia, dysmetria and rest tremor of the head. Tendon reflexes and muscle power are weak. In addition, he also suffers from episodes of diarrhea and vomiting of unknown cause. An MRI scan, performed
4 years ago, did show cerebellar atrophy, however, the typical lesions were not obserevd. This patient was known as a normal transmitting male to the clinicians. He was found to carry 111 repeats. The fourth patient has a very similar clinical history, with onset of progressive ataxia at the age of 70 years. Subsequently, this patient developed an intention tremor and dysarthria, together with intellectual failing. His MRI at the age of 80 years showed the typical cerebellar lesions together with cortical and subcortical atrophy. At his last evaluation, he was wheelchair bound, depressed and severely cognitive impaired. He died 3 years ago, at the age of 86 years. This patient was shown to have 82 repeats. Patient 5 reported onset of walking problems at the age of 67 years. Subsequently, mild bilateral intention tremor was noted. MRI scan at that time showed the typical cerebellar lesions, periventricular leucomalacia, dilated ventricles and diffuse cortical athrophy. He died 2 years later due to an intestinal adenocarcinoma. This patient was shown to carry 84 repeats.

All patients were seen in the past by different neurologists and during their follow-up different diagnoses were proposed, including Parkinson disease, olivo-ponto-cereballar-atrophy, gluten-ataxia, paraneoplastic phenomenon and Alzheimer dementia. In four, a definite diagnosis of FXTAS can be made, based on the proposed diagnostic clinical and radiological criteria for FXTAS. Patient 3 did not show the cerebellar lesions on MRI performed 4 years ago. Unfortunately, imaging could not be repeated because of his clinical deterioration. However, given the typical clinical features present in this patient, the diagnosis of FXTAS is probable. Interestingly, the clinical course of the disease is very similar in our group of patients, with presence of the specific cerebellar white matter lesions in four of them. In the youngest patient (patient 2), with recent onset of very mild ataxia and discrete tremor, these specific radiological findings are already present. Further follow-up of this patient is needed to document progression. The mean age at onset of symptoms in our FXTAS patients is 64 years 9 months, which is in agreement with the recent figures of Jacquemont et al. ${ }^{2}$

The repeat sizes observed in our patients range from 80 to 111 (mean: 90). Thus far no correlations could be made between onset/progression-rate and repeat size because of ascertainment bias in most large studies. In previous screens of older adult males presenting with ataxia, repeat lengths of 51,66, 87, of 80 and of 84,86 have been found, respectively, by Macpherson et al, ${ }^{4}$ Milunsky and Maher, ${ }^{5}$ and Di Maria et al. ${ }^{6}$ Although it is currently not proven that these FRAXA repeats $>50$ are causative in all these reported cases, the repeat range found via this approach is in concordance with the mean repeat length reported in the fragile X family-based studies. ${ }^{7}$ At present, the smallest repeat size which can give rise to FXTAS is not known. In our cohort of 122 males, we identified two males within the repeat range of 45-50. When compared with the 
results of an FMR-1 screening conducted in a population of 2534 control X chromosomes obtained from a survey of normal pregnant Flemish females (E Legius and G Matthijs, unpublished data), this number falls within what is expected (two-sided mid-P value: 0.23 , Fishers exact test). However, the present study design does not allow us to answer the question if the repeat sizes within the gray zone are indeed related to the development of FXTAS and further prospective studies are warranted.

This study represents the first large Flemish cohort of older male patients, negative for $S C A 1,2,3,6$ and 7, that were screened for the FMR-1 premutation. Of this cohort, $4.1 \%$ were found to be premutated, confirming the observation that $\pm 5 \%$ of cases of sporadic ataxia in older males may be attributable to the effects of premutation alleles. The prevalence observed in the present study is in line with the figures reported by MacPherson et $a l^{4}$, who found three of 59 males carrying the premutation. The lower prevalence detected by Milunsky et $a l,{ }^{5}$ who detected only one out of 167 males with a premutation, is most likely due to a difference in referral and sample population. On the total group of 137 referred ataxia patients, the premutated group represents 3.6 versus $10.9 \%$ of the SCApositive group. However, when we evaluate each SCA gene separately we found 8/137 (5.8\%) males with an SCA6 expansion, 3/137 (2.2\%) with an SCA7 expansion, another $3 / 137(2.2 \%)$ with an SCA2 expansion, and one patient with an expansion in the SCA1 gene (0.7\%). In light of these figures, we recommend that FMR-1 analysis is included in the group of male ataxia patients older than 50 years, especially when additional symptoms such as tremor, parkinsonism and cognitive decline are present. However, this raises also important ethical and counseling issues. Since most of these elder men may already have children, inclusion of FMR-1 in routine screening can only be offered when genetic counseling towards the individual and his family is provided. Based on the current data, in our centre, we systematically offer to the referring physician FMR-1 testing in all male ataxia patients older than 50 years and negative for SCA, after consent of the patient.

\section{Acknowledgements}

HVE is a post-doctoral researcher of the Fund for Scientific ResearchFlanders, Belgium (FWO-Vlaanderen). This work was supported by the European Union RTD Project No. QLRT-2001-01810.

\section{References}

1 Hagerman RJ, Leehey M, Heinrichs $\mathrm{W}$ et al: Intention tremor, parkinsonism, and generalized brain atrophy in male carriers of fragile X. Neurology 2001; 57: 127-130.

2 Jacquemont S, Hagerman RJ, Leehey $\mathrm{M}$ et al: Fragile X premutation tremor/ataxia syndrome: molecular, clinical, and neuroimaging correlates. Am J Hum Genet 2003; 72: 869-878.

3 Jacquemont S, Hagerman RJ, Leehey MA et al: Penetrance of the fragile $\mathrm{X}$-associated tremor/ataxia syndrome in a premutation carrier population. JAMA 2004; 291: 460-469.

4 Macpherson J, Waghorn A, Hammans S, Jacobs P: Observation of an excess of fragile-X premutations in a population of males referred with spinocerebellar ataxia. Hum Genet 2003; 112: 619-620.

5 Milunsky JM, Maher TA: Fragile $X$ carrier screening and spinocerebellar ataxia in older males. Am J Med Genet 2004; 125A: 320 .

6 Di Maria E, Grasso M, Pigullo S et al: Further evidence that a tremor:ataxia syndrome may occur in fragile $\mathrm{X}$ premutation carriers. Paper presented at the American Society of Human Genetics, 53rd Annual Meeting, Los Angeles, 4-8 November 2003.

7 Jacquemont S, Farzin F, Hall D et al: Aging in individuals with the FMR1 mutation. Am J Ment Retard 2004; 109: 154-164. 